\title{
recillunds
}

Revista Científica Mundo de la Investigación y el Conocimiento

Denisse Johanna Romero Lorenti a ; Rudy Stefani Vargas López ${ }^{\text {b; }}$ Karla Estefanía

López Olives ${ }^{c}$; Evelyn Ileana Reyes Yagual ${ }^{\mathrm{d}}$

Fisiopatología y riesgo materno-fetal asociados a la preeclampsia

Physiopathology and maternal-fetal risk associated with preeclampsy

Revista Científica Mundo de la Investigación y el Conocimiento. Vol. 3 núm.3. Esp., noviembre, ISSN: 2588-073X, 2019, pp. 589-606

DOI: $10.26820 /$ recimundo/3.(3.Esp).noviembre.2019.589-606

URL: http://recimundo.com/index.php/es/article/view/612

Código UNESCO: 3205 Medicina Interna

Tipo de Investigación: Artículo de Revisión

(ㄱ) RECIMUNDO; Editorial Saberes del Conocimiento, 2019

Recibido: 15/09/2019

Aceptado: 23/10/2019

Publicado: 30/11/2019

Correspondencia: denisseromero2710@gmail.com

\footnotetext{
a. Médico; Investigadora Independiente; Guayaquil, Ecuador; denisseromero2710@ gmail.com

b. Médico; Investigadora Independiente; Guayaquil, Ecuador; rudy_vargas_1@ hotmail.com

c. Médico; Investigadora Independiente; Guayaquil, Ecuador; gussy20k@ hotmail.com

d. Médico; Investigadora Independiente; Guayaquil, Ecuador; velynry@ gmail.com
} 


\section{Fisiopatología y riesgo materno-fetal asociados a la preeclampsia}

Vol. 3, núm. 3 Esp., (2019)

Denisse Johanna Romero Lorenti; Rudy Stefani Vargas López; Karla Estefanía López Olives;

Evelyn Ileana Reyes Yagual

\section{RESUMEN}

Mediante la realización de este trabajo se busca identificar los factores de riesgo epidemiológicos que producen preeclampsia en las mujeres embarazadas, a través de una revisión bibliográfica de casos publicados que proporcionan información importante para el desarrollo de la investigación. La preeclampsia se caracteriza por la aparición de hipertensión y disfunción del órgano terminal incluyendo proteinuria después de 20 semanas de gestación. Esta patología generalmente se presenta durante el embarazo, sin embargo, a veces puede manifestarse en el período posparto en una mujer previamente normotensa. Los factores identificados con mayor frecuencia son mujeres con antecedentes de preeclampsia, preexistentes, diabetes, embarazo múltiple (gemelo), nuliparidad, antecedentes familiares, presión arterial elevada (diastólica $\geq 80 \mathrm{~mm} \mathrm{Hg}$ ) al momento de la reserva, aumento del índice de masa corporal antes del embarazo o aumento de la edad materna $\geq 40$. En algunos casos, el riesgo también aumenta con el tiempo de espera entre embarazo y embarazo con intervalos de 10 años o más desde un embarazo anterior, además de la presencia de enfermedades autoinmune, enfermedades renales e hipertensión crónica.

Palabras Claves: Preeclampsia; Factores de riesgo; Presión arterial; Embarazo; Hipertensión. 


\title{
Fisiopatología y riesgo materno-fetal asociados a la preeclampsia
}

Vol. 3, núm. 3 Esp., (2019)

Denisse Johanna Romero Lorenti; Rudy Stefani Vargas López; Karla Estefanía López Olives;

Evelyn Ileana Reyes Yagual

\begin{abstract}
By carrying out this work, we seek to identify the epidemiological risk factors that produce preeclampsia in pregnant women, through a bibliographic review of published cases that require important information for the development of the research. Preeclampsia is characterized by the appearance of hypertension and dysfunction of the terminal organ including proteinuria after 20 weeks of gestation. This pathology usually occurs during pregnancy, however, it can sometimes manifest itself in the postpartum period in a previously normotensive woman. The most frequently identified factors are women with a history of preeclampsia, preexisting, diabetes, multiple pregnancy (twin), nulliparity, family history, high blood pressure (diastolic $\geq 80 \mathrm{~mm}$ $\mathrm{Hg}$ ) at the time of reservation, increase in body mass index before pregnancy or maternal age increase $\geq 40$. In some cases, the risk also increases with the waiting time between pregnancy and pregnancy with intervals of 10 years or more from a previous pregnancy, in addition to the presence of autoimmune diseases, diseases renal and chronic hypertension.
\end{abstract}

Key Worda: Preeclampsia; Risk factors; Blood pressure; Pregnancy; Hypertension. 


\section{Fisiopatología y riesgo materno-fetal asociados a la preeclampsia}

Vol. 3, núm. 3 Esp., (2019)

Denisse Johanna Romero Lorenti; Rudy Stefani Vargas López; Karla Estefanía López Olives;

Evelyn Ileana Reyes Yagual

\section{Introducción.}

Los trastornos hipertensivos del embarazo son uno de las causas más comunes de morbilidad materna y fetal con un índice de mortalidad de más de 40.000 muertes maternas en todo el mundo anualmente. En los países desarrollos, causa aproximadamente un tercio de la mortalidad materna.

Un estudio realizado por el Ministerio de Salud y Bienestar en el año 2013 en India representó, que en ese país el índice de mortalidad representa el 19\% de las muertes maternas en todo el mundo. Cinco por ciento de las muertes maternas en India se deben al trastorno hipertensivo. (Ramesh, Gandhi, \& Rao, 2014). La preeclampsia (PE) ha seguido siendo una amenaza significativa para la salud en los países desarrollados y en desarrollo, sin embargo, el impacto de la enfermedad es mayor en los países en desarrollo, donde el tratamiento puede ser ineficaz debido a la presentación tardía de los casos.

Aunque la preeclampsia no es totalmente prevenible, su detección temprana y el tratamiento adecuado puede prevenir la gravedad, su etiología poco clara y lo impredecible, ya que, la naturaleza de la enfermedad empeora aún más la situación. La incidencia de PE varía del $2 \%$ al $10 \%$. La OMS estima que la incidencia de preeclampsia es siete veces mayor en los países en desarrollo (2.8\% de nacimientos vivos) que en los países desarrollados $(0.4 \%)$. (Endeshaw, Ambaw, Aragaw, \& Ayalew, 2014).

La preeclampsia se ha asociado con un mayor riesgo de resultados adversos fetales, neonatales y maternos que incluyen hemorragia preparto y posparto, insuficiencia renal y hepática aguda, convulsiones eclámpticas, accidente cerebrovascular, desprendimiento de la 


\section{Fisiopatología y riesgo materno-fetal asociados a la preeclampsia}

Vol. 3, núm. 3 Esp., (2019)

Denisse Johanna Romero Lorenti; Rudy Stefani Vargas López; Karla Estefanía López Olives;

Evelyn Ileana Reyes Yagual

placenta, síndrome HELLP, falla en el corazón, fallas de múltiples órganos y muerte materna. (North, y otros, 2011). Las complicaciones fetales incluyen sufrimiento fetal, retraso del crecimiento intrauterino, parto prematuro, muerte fetal, muerte perinatal y asfixia neonatal.

Los factores que se han encontrado en la influencia del riesgo de preeclampsia en varios estudios incluyen diabetes y diabetes gestacional, obesidad, embarazos múltiples, antecedentes personales y familiares de preeclampsia, infecciones crónicas, ITU, primer embarazo y edad materna menor de 20 años edad, enfermedad renal y trastorno autoinmune, intervalo prolongado entre embarazos e historia del aborto y la dieta materna. (Minassian, Thomas, Williams, Campbell, \& Smeeth, 2013).

El conocimiento e identificación de los aspectos y factores de riesgo clínico para la EP ayudarán a estimar el riesgo individualizado de cada mujer permitiendo que la vigilancia prenatal se dirija a estas mujeres para guiar a los proveedores de atención médica en el asesoramiento y posiblemente reducir la recurrencia de riesgo de EP, si algunos factores de riesgo modificables (como obesidad) están presentes.

Si bien la mayoría de los estudios se han realizado en países de altos ingresos ajustes, existen algunas inconsistencias, especialmente países en desarrollo donde los factores de riesgo de preeclampsia han sido menos explorados. Además, las condiciones como la anemia severa y la falta de atención prenatal que son más frecuentes en las regiones menos desarrolladas requieren más investigación y validación de hallazgos. 


\section{Fisiopatología y riesgo materno-fetal asociados a la preeclampsia}

Vol. 3, núm. 3 Esp., (2019)

Denisse Johanna Romero Lorenti; Rudy Stefani Vargas López; Karla Estefanía López Olives;

Evelyn Ileana Reyes Yagual

\section{Métodos y Materiales.}

Para el desarrollo de este proceso investigativo, se plantea como metodología la encaminada hacia una orientación científica particular que se encuentra determinada por la necesidad de indagar en forma precisa y coherente una situación, en tal sentido (Davila, 2015) define la metodología "como aquellos pasos previos que son seleccionados por el investigador para lograr resultados favorables que le ayuden a plantear nuevas ideas".(p.66).

Lo citado por el autor, lleva a entender que el desarrollo de la acción investigativa busca simplemente coordinar acciones enmarcadas en una revisión bibliográfica con el fin de complementar ideas previas relacionadas Fisiopatología y riesgo materno-fetal asociados a la preeclampsia a través de una revisión de literatura, para así finalmente elaborar un cuerpo de consideraciones generales que ayuden a ampliar el interés propuesto.

\section{Tipo de Investigación}

Dentro de toda práctica investigativa, se precisan acciones de carácter metodológico mediante las cuales, se logra conocer y proyectar los eventos posibles que la determinan, así como las características que hacen del acto científico un proceso interactivo ajustado a una realidad posible de ser interpretada. En este sentido, se puede decir, que la presente investigación corresponde al tipo documental, definido por Castro (2016), "se ocupa del estudio de problemas planteados a nivel teórico, la información requerida para abordarlos se encuentra básicamente en materiales impresos, audiovisuales y /o electrónicos”. (p.41). 


\section{Fisiopatología y riesgo materno-fetal asociados a la preeclampsia}

Vol. 3, núm. 3 Esp., (2019)

Denisse Johanna Romero Lorenti; Rudy Stefani Vargas López; Karla Estefanía López Olives;

Evelyn Ileana Reyes Yagual

En consideración a esta definición, la orientación metodológica permitió la oportunidad de cumplir con una serie de actividades inherentes a la revisión y lectura de diversos documentos donde se encontraron ideas explicitas relacionadas con los tópicos encargados de identificar a cada característica insertada en el estudio. Por lo tanto, se realizaron continuas interpretaciones con el claro propósito de revisar aquellas apreciaciones o investigaciones propuestas por diferentes investigadores relacionadas con el tema de interés, para luego dar la respectiva argumentación a los planteamientos, en función a las necesidades encontradas en la indagación.

\section{Fuentes Documentales}

El análisis correspondiente a las características que predomina en el tema seleccionado, llevan a incluir diferentes fuentes documentales encargadas de darle el respectivo apoyo y en ese sentido cumplir con la valoración de los hechos a fin de generar nuevos criterios que sirven de referencia a otros procesos investigativos. Para (CASTRO, 2016) las fuentes documentales incorporadas en la investigación documental o bibliográfica, "representa la suma de materiales sistemáticos que son revisados en forma rigurosa y profunda para llegar a un análisis del fenómeno".(p.41). Por lo tanto, se procedió a cumplir con la realización de una lectura previa determinada para encontrar aquellos aspectos estrechamente vinculados con el tema, con el fin de explicar mediante un desarrollo las respectivas apreciaciones generales de importancia.

\section{Técnicas para la Recolección de la Información}

La conducción de la investigación para ser realizada en función a las particularidades que determinan a los estudios documentales, tiene como fin el desarrollo de un conjunto de acciones encargadas de llevar a la selección de técnicas estrechamente vinculadas con las características 


\section{Fisiopatología y riesgo materno-fetal asociados a la preeclampsia}

Vol. 3, núm. 3 Esp., (2019)

Denisse Johanna Romero Lorenti; Rudy Stefani Vargas López; Karla Estefanía López Olives;

Evelyn Ileana Reyes Yagual

del estudio. En tal sentido, (Bolívar, 2015), refiere, que es "una técnica particular para aportar ayuda a los procedimientos de selección de las ideas primarias y secundarias”. (p. 71).

Por ello, se procedió a la utilización del subrayado, resúmenes, fichaje, como parte básica para la revisión y selección de los documentos que presentan el contenido teórico. Es decir, que mediante la aplicación de estas técnicas se pudo llegar a recoger informaciones en cuanto a la revisión bibliográfica de los diversos elementos encargados de orientar el proceso de investigación. Tal como lo expresa, (Bolívar, 2015) "las técnicas documentales proporcionan las herramientas esenciales y determinantes para responder a los objetivos formulados y llegar a resultados efectivos" (p. 58). Es decir, para responder con eficiencia a las necesidades investigativas, se introdujeron como técnica de recolección el método inductivo, que hizo posible llevar a cabo una valoración de los hechos de forma particular para llegar a la explicación desde una visión general.

Asimismo, se emplearon las técnicas de análisis de información para la realización de la investigación que fue ejecutada bajo la dinámica de aplicar diversos elementos encargados de determinar el camino a recorrer por el estudio, según, (Bolívar, 2015) las técnicas de procesamiento de datos en los estudios documentales "son las encargadas de ofrecer al investigador la visión o pasos que debe cumplir durante su ejercicio, cada una de ellas debe estar en correspondencia con el nivel a emplear" (p. 123). Esto indica, que para llevar a cabo el procesamiento de los datos obtenidos una vez aplicado las técnicas seleccionadas, tales como: fichas de resumen, textual, registros descriptivos entre otros, los mismos se deben ajustar al nivel que ha sido seleccionado. 


\section{Fisiopatología y riesgo materno-fetal asociados a la preeclampsia}

Vol. 3, núm. 3 Esp., (2019)

Denisse Johanna Romero Lorenti; Rudy Stefani Vargas López; Karla Estefanía López Olives;

Evelyn Ileana Reyes Yagual

\section{Resultados.}

La fisiopatología exacta de la preeclampsia permanece desconocida. Sin embargo, hay varias teorías que se han presentado que pueden explicar la mayoría de las anormalidades visto en este proceso de la enfermedad.

\section{Placentación anormal}

La preeclampsia es principalmente una enfermedad de la placenta como puede encontrarse en embarazos molares. Uno de las teorías más aceptadas en preeclampsia gira alrededor de placentación anormal. En embarazos normales, el trofoblasto comienza la invasión en la sangre a través del vaso miometrio mediante la remodelación de las arterias espirales maternas, transformandolos de pequeño a musculoso con mayor resistencia arteriolas en arterias de gran calibre con alta capacidad y libre flujo de sangre. (Lim, Zhou, \& Janatpour, 2010).

La remodelación típicamente comienza a fines del primer trimestre y se completa a las 18-20 semanas de gestación. El fracaso de este proceso de completar la remodelación conduce a la persistencia de alta resistencia de arterias espirales que impiden la perfusión placentaria lo que conduce a un estado de "hipoxemia relativa que culmina con la disfunción de células endoteliales maternas. La disfunción sistémica materna de células endoteliales se manifiesta en signos y síntomas que reflejan la maternidad vasoconstricción y daño multiorgánico como se observa esbozado en la tabla $\mathrm{N}^{\circ} 1$. 
Fisiopatología y riesgo materno-fetal asociados a la preeclampsia

Vol. 3, núm. 3 Esp., (2019)

Denisse Johanna Romero Lorenti; Rudy Stefani Vargas López; Karla Estefanía López Olives;

Evelyn Ileana Reyes Yagual

Tabla 1: Disfunción de órganos terminales en preeclampsia.

\begin{tabular}{|c|l|}
\hline Trombocitopenia & Recuento de plaquetas $<100,000 /$ micro \\
\hline Disfunción hepática & AST o ALT $>2$ veces el límite superior de lo normal \\
\hline Insuficiencia renal & Creatinina $>1.1 \mathrm{mg} / \mathrm{dL}$ o duplicación de la creatinina basal \\
\hline Edema pulmonar & \\
\hline Trastornos del sistema nervioso & Dolor de cabeza severo, escotomas, estado mental alterado \\
\hline central & \\
\hline
\end{tabular}

Fuente: (Colegio Americano de Obstetras y Ginecólogos, 2013).

La hipoperfusión placentaria es tanto una causa como un efecto de la placentación anormal que se vuelve más pronunciado con las crecientes necesidades del feto-placentario a medida que avanza el embarazo. Los cambios patológicos tardíos que se ven en el tejido placentario se correlacionan con la isquemia incluyendo aterosis, necrosis fibrinoide, trombosis, esclerosis de las arteriolas e infarto. (Maqueo \& Chavezazuela, 2007). 


\section{Fisiopatología y riesgo materno-fetal asociados a la preeclampsia}

Vol. 3, núm. 3 Esp., (2019)

Denisse Johanna Romero Lorenti; Rudy Stefani Vargas López; Karla Estefanía López Olives;

Evelyn Ileana Reyes Yagual

\section{Factores inmunológicos}

La preeclampsia tiende a afectar el primer embarazo (ingenuo para los antígenos paternos fetales) y exposición repetida a los antígenos paternos fetales tiende a disminuir el riesgo de la enfermedad. Además, existen otras situaciones que limitan la exposición a antígenos paternos, como una nueva pareja en un embarazo posterior o intervaos largos entre un embarazo y otro, el uso de anticonceptivos de barrera o concepción a través de la inseminación artificial tambien conducen a un mayor riesgo de preeclampsia. También se sabe que las mujeres que conciben a través de la donación de óvulos tienen más del doble de riesgo de preeclampsia que otras formas de reproducción asistida. (Blazquez, Garcia, Rodriguez, \& Vassena, 2016). En los embarazos concebidos a través de asistencia, las técnicas reproductivas tuvieron un aumento de cuatro veces en la preeclampsia en comparación con embarazos concebidos naturalmente.

Las anomalías observadas en la preeclampsia son similares a las observados en la enfermedad de injerto contra huésped. La interacción entre los antígenos extraños del trofoblasto velloso y las células asesinas naturales dictan la implantación placentaria. En preeclampsia, la implantación placentaria anormal se cree que se debe al aumento de la actividad de las células NK debido al conflicto entre los genes maternos y paternos. Esto puede estar mediado en parte a través de la célula dendrítica en la actividad como el aumento de la infiltración de células dendríticas que se ve en biopsias de lecho placentario con preeclampsia. (LaMarca \& Ryan, 2007). 


\section{Fisiopatología y riesgo materno-fetal asociados a la preeclampsia}

Vol. 3, núm. 3 Esp., (2019)

Denisse Johanna Romero Lorenti; Rudy Stefani Vargas López; Karla Estefanía López Olives;

Evelyn Ileana Reyes Yagual

\section{Inflamación y / o infección}

Se observan signos de inflamación en embarazos normales a término, pero estos cambios son exagerados en las mujeres con preeclampsia Se cree que los restos del exterior de la capa de trofoblasto contribuye a la inflamación materna y algunas características del síndrome. El ADN placentario liberado en la circulación de la madre y el feto (circulación materna) podría tener un papel en la inflamación característica involucrada con preeclampsia. En estudios que analizaron la conexión entre la infección materna y la preeclampsia, se encontró que el riesgo de preeclampsia aumentó en mujeres con infección del tracto urinario y enfermedad periodontal. (Haggerty \& Klebanoff, 2013).

\section{Factores genéticos}

Se cree que los factores genéticos tienen un papel importante en la enfermedad. Las mujeres que están embarazadas por primera vez y tienen antecedentes familiares de preeclampsia tienen un mayor riesgo de contraer que las mujeres que están embarazadas por primera sin tener antecedentes familiares de preeclampsia. El riesgo de preeclampsia aumenta significativamente en mujeres que previamente tenía preeclampsia. Las parejas de hombres cuyas madres que tuvieron preeclampsia tambien tienen más probabilidades de que sus parejas presenten preeclampsia. La mayoría de los datos sugieren que la madre y los genes del padre tienen un papel en la formación defectuosa de la placenta y posterior preeclampsia. (Dekker \& Robillard, 2011).

En resumen, la hipoxia placentaria y la isquemia son las últimas vías en la patogénesis de la preeclampsia mediante la liberación de factores vasoactivos en la circulación materna y la 


\section{Fisiopatología y riesgo materno-fetal asociados a la preeclampsia}

Vol. 3, núm. 3 Esp., (2019)

Denisse Johanna Romero Lorenti; Rudy Stefani Vargas López; Karla Estefanía López Olives;

Evelyn Ileana Reyes Yagual

disfunción de las células endoteliales que conduce a los signos y síntomas de preeclampsia. (Dekker \& Robillard, 2011).

¿Cuáles son los factores de riesgo para la preeclampsia?

Existen varios factores de riesgo bien estudiados para la preeclampsia y la magnitud del riesgo depende de el factor individual, la gravedad y la cantidad de riesgo los factores se muestran en la (tabla 2).

Tabla 2: Factores de riesgo para preeclampsia.

\begin{tabular}{|l|c|}
\hline 1 & Primer embarazo \\
\hline 2 & Preeclampsia previa \\
\hline 3 & Hipertensión crónica \\
\hline 4 & Obesidad \\
\hline 5 & Enfermedad renal crónica \\
\hline 6 & Historia de trombofilia. \\
\hline 7 & Gestación múltiple \\
\hline
\end{tabular}


Fisiopatología y riesgo materno-fetal asociados a la preeclampsia

Vol. 3, núm. 3 Esp., (2019)

Denisse Johanna Romero Lorenti; Rudy Stefani Vargas López; Karla Estefanía López Olives; Evelyn Ileana Reyes Yagual

\begin{tabular}{|c|c|}
\hline 8 & Técnicas de reproducción asistida \\
\hline 9 & Antecedentes familiares de preeclampsia. \\
\hline 10 & Diabetes mellitus tipo I o tipo II \\
\hline 11 & Obesidad \\
\hline 12 & Edad $>40$ \\
\hline 13 & Intervalo de embarazo prolongado \\
\hline 14 & Raza afroamericana \\
\hline 15 & Embarazo molar \\
\hline
\end{tabular}

Fuente: (Pare \& Parry, 2011).

El mayor riesgo es el antifosfolípido materno síndrome de anticuerpos: un aumento de nueve veces riesgo de desarrollar preeclampsia seguido de antecedentes de preeclampsia en un embarazo anterior que confiere un séptuplo aumento del riesgo. Además, la mayor gravedad de preeclampsia en el embarazo anterior se asocia con un mayor riesgo de preeclampsia en la 


\section{Fisiopatología y riesgo materno-fetal asociados a la preeclampsia}

Vol. 3, núm. 3 Esp., (2019)

Denisse Johanna Romero Lorenti; Rudy Stefani Vargas López; Karla Estefanía López Olives;

Evelyn Ileana Reyes Yagual

gestación posterior. (Pare \& Parry, 2011). Otros factores incluyen diabetes, hipertensión, gestación múltiple, antecedentes afroamericanos, asistida reproducción y obesidad.

¿Se puede prevenir la preeclampsia?

Dado el riesgo para la madre y el feto, se han intentado numerosas intervenciones para prevenir la preeclampsia pero desafortunadamente ninguno ha demostrado ser útil para la prevencion primaria de preeclampsia. En un subconjunto de mujeres con un embarazo previo complicado por preeclampsia, el uso de dosis bajas de aspirina es beneficiosa.

En un metanálisis (Lynch \& McDuffie, 2002) demostraron una reducción del riesgo del $17 \%$ en la preeclampsia con el uso de agentes antiplaquetarios con una significativa disminución en la reducción del riesgo absoluto en mujeres con alto riesgo de enfermedad. Durante la investigacion sobre hipertensión en el embarazo se recomiendó iniciar una dosis baja de aspirina a finales del primer trimestre para mujeres con antecedentes de preeclampsia con parto posterior a los 34 años o antes con semanas de gestación.

\section{Conclusiones.}

Durante el desarrollo de la investigación se ha observado que la preeclampsia es un nuevo inicio de hipertensión a lo largo del embarazo con proteinuria después de 20 semanas de gestación que puede estar asociado con disfunciones de múltiples órganos. Sin embargo, la etiología precisa de la preeclampsia sigue siendo desconocida. Varias teorías han presentado que la preeclampsia afecta tanto a la madre como al feto, siendo una de las causas más importantes de morbilidad materna e incluso mortalidad. 


\section{Fisiopatología y riesgo materno-fetal asociados a la preeclampsia}

Vol. 3, núm. 3 Esp., (2019)

Denisse Johanna Romero Lorenti; Rudy Stefani Vargas López; Karla Estefanía López Olives;

Evelyn Ileana Reyes Yagual

La preeclampsia aumenta el riesgo de padecer nuevamente la enfermedad en un embarazo

posterior. Las mujeres con preeclampsia en un primer embarazo desarrollarán embarazos con hipertensión y serán diagnosticados con preeclampsia en embarazos posteriores.

Es importante que para mujeres con factores de riesgo de preeclampsia se tenga un asesoramiento previo a la concepción, ya que, esto les proporciona una oportunidad para identificar riesgos potencialmente modificables, garantizando una evaluación completa y más a fondo y una historia física que conlleve a la realización de exámenes y pruebas de laboratorio pertinentes a tiempo temprano en el embarazo que puede así establecer una línea de base y comparar la edad gestacional. Las mujeres deberían conocer los signos comunes y los síntomas de preeclampsia y ser monitoreadas de cerca por el proceso de la enfermedad. Las mujeres también deben ser informadas del aumento riesgo de enfermedad cardiovascular futura.

Tomando en consideración que esta enfermedad afecta tanto a la madre como al feto se debe tener en cuenta que la presencia de preeclampsia ocasiona una perfusión placentaria comprometida que conduce a una disminución en el flujo sanguíneo al feto en desarrollo. Así como también complicaciones fetales que incluyen parto prematuro, restricción del crecimiento fetal y desprendimiento placentario. Sin embargo, las complicaciones maternas están relacionados principalmente con el daño del sistema de órganos, incluyendo edema pulmonar, convulsiones, insuficiencia renal, hígado hematoma o ruptura, complicaciones hemorrágicas, entre otros. 


\section{Fisiopatología y riesgo materno-fetal asociados a la preeclampsia}

Vol. 3, núm. 3 Esp., (2019)

Denisse Johanna Romero Lorenti; Rudy Stefani Vargas López; Karla Estefanía López Olives;

Evelyn Ileana Reyes Yagual

\section{Bibliografía.}

Blazquez, Garcia, Rodriguez, \& Vassena. (2016). ¿Es la donación de ovocitos un factor de riesgo para la preeclampsia? Una revisión sistemática y un metanálisis. . (págs. 855-863.). Bogota: Reprod Genet.

Bolívar, J. (2015). Investigación Documental. México: Pax.

Castro, J. (2016). Técnicas Documentales. México: Limusa.

Davila, A. (2015). Diccionario de Términos Científicos. Caracas: Editorial Oasis.

Dekker, G., \& Robillard, P. (2011). The etiology of preeclampsia: the role of the father. 89(2), $126-132$.

Endeshaw, Ambaw, Aragaw, \& Ayalew. (2014). Effect of Maternal Nutrition and Dietary Habits on Preeclampsia: A Case-Control Study. International Journal of Clinical Medicine, 5(21), 1405-1416.

Haggerty, \& Klebanoff. (2013). Prenatal Chlamydia trachomatis infection increases the risk of preeclampsia. Pregnancy Hypertens. Pregnancy Hypertens, 3(3), 151-198.

LaMarca, B., \& Ryan, M. (2007). Citocinas inflamatorias en la fisiopatología de la hipertensión durante la preeclampsia. . Mexico: Curr Hypertens.

Lim, Zhou, \& Janatpour. (2010). La diferenciación / invasión de citotrofoblasto humano es anormal en preeclampsia. Buenos Aires: Am J Pathol.

Lynch, A., \& McDuffie, R. (2002). Preeclampsia en gestación múltiple: el papel de las tecnologías de reproducción asistida . 99-167: Obstet Gynecol.

Maqueo, M., \& Chavezazuela, J. (2007). Patología placentaria en eclampsia y preeclampsia . Obstet Gynecol.

Minassian, C., Thomas , S. L., Williams , D. J., Campbell, O., \& Smeeth, L. (2013). Infección materna aguda y riesgo de preeclampsia. PLoS One, 8(9), 1-8.

North, R. A., McCowan, L. M., Dekker, G. A., Poston, L., Chan, E. H., Stewart, A. W., . . . Kenny, L. C. (2011). Predicción de riesgo clínico para preeclampsia en mujeres nulíparas: desarrollo de modelo en cohorte prospectiva internacional. BMJ, 342, 1-11.

Pare, E., \& Parry, S. (2011). Clinical risk factors for preeclampsia in the 21st century. Obstet Gynecol, 124(4), 763-770. 
Fisiopatología y riesgo materno-fetal asociados a la preeclampsia

Vol. 3, núm. 3 Esp., (2019)

Denisse Johanna Romero Lorenti; Rudy Stefani Vargas López; Karla Estefanía López Olives;

Evelyn Ileana Reyes Yagual

Ramesh, Gandhi, \& Rao. (2014). Socio-Demographic and Other Risk Factors of Pre Eclampsia at a Tertiary Care Hospital, Karnataka: Case Control Study. J Clin Diagn Res, 8(9).

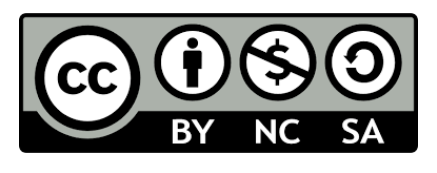

RECONOCIMIENTO-NOCOMERCIAL-COMPARTIRIGUAL

CC BY-NC-SA

ESTA LICENCIA PERMITE A OTROS ENTREMEZCLAR, AJUSTAR Y CONSTRUIR A PARTIR DE SU OBRA CON FINES NO

COMERCIALES, SIEMPRE Y CUANDO LE RECONOZCAN LA AUTORÍA Y SUS NUEVAS CREACIONES ESTÉN BAJO UNA LICENCIA CON LOS MISMOS TÉRMINOS. 